\title{
Buckling of a thin elastic rod inside a horizontal cylindrical constraint
}

\author{
Jay T. Miller ${ }^{\mathrm{a}, 1}$, Tianxiang Su ${ }^{\mathrm{b}}$, Jahir Pabon ${ }^{\mathrm{b}}$, Nathan Wicks ${ }^{\mathrm{b}}$, Katia Bertoldic,d, Pedro M. Reis ${ }^{\mathrm{a}, \mathrm{e}, *}$ \\ ${ }^{a}$ Dept. of Civil and Environmental Engineering, Massachusetts Institute of Technology, Cambridge, MA 02139, USA \\ ${ }^{b}$ Schlumberger-Doll Research, One Hampshire Street, Cambridge, MA 02139, USA \\ ${ }^{c}$ School of Engineering and Applied Sciences, Harvard University, Cambridge, MA 02138, USA \\ ${ }^{d}$ Kavli Institute for Bionano Science and Technology, Harvard University, Cambridge, MA 02138, USA \\ ${ }^{e}$ Dept. Mechanical Engineering, Massachusetts Institute of Technology, Cambridge, MA 02139, USA
}

\begin{abstract}
We present results from an experimental and numerical investigation on the compression, and consequent buckling, of a slender rod constrained inside a horizontal cylinder. An experimental model system is developed to systematically study the sequence of instabilities from straight-to-sinusoidal and sinusoidal-to-helical configurations. We quantify the associated buckling loads as a function of the radial clearance between the rod and cylindrical constraint. These results are compared to existing theoretical predictions. While good agreement is obtained for large values of the radial clearance, significant deviations are found when the geometric imperfections of the setup are comparable to the radial clearance. Due to this imperfection sensitivity, the critical buckling loads can be reduced significantly by up to a factor of three. The findings from this model system can be applied to practical applications across a range of length scales due to the geometric (rather than material) nonlinearities involved in the deformations of rods.
\end{abstract}

Keywords: thin elastic rods, buckling, geometric nonlinearities

\section{Introduction}

Buckling of slender rodlike structures under lateral confinement can lead to complex geometric nonlinearities and is ubiquitous in both natural and engineered settings, across $_{30}$ 5 a wide range of length scales. Examples include DNA packings inside viral capsules [1, silicon nanowires attached to stretchable substrates [2, jamming of nanorods confined inside a channel [3, plant roots penetrating into substrates that have a graded stifness [4] and kilometerlong pipes inside of a borehole in oil well operations [5, 6.

In the context of drilling, the buckling of a rod inside a vertical cylinder was first investigated by Lubinski et al. 7, 5], using equilibrium methods and neglecting the effect of friction. For the case of an inclined, or horizon15 tal, cylindrical constraint, past a first critical load, $P_{s}$, the rod takes on a sinusoidal configuration, with a well defined wavelength, $\lambda_{c}^{s}$. Lateral deflections of the buckled rod are penalized by the combined effect of elastic and gravitational potential energies, which makes higher ${ }_{45}$ buckling modes more energetically favorable than the first mode. Following energy methods, expressions for $P_{s}$ and $\lambda_{c}^{s}$ were derived by Paslay and Bogey [6] and Dawson and Paslay [8], respectively. Further compression past a second critical load, $P_{h}$, results in the rod transitioning into a heli-

\footnotetext{
* Corresponding author

Email address: preis@mit.edu (Pedro M. Reis)

${ }^{1}$ Now a Research Associate with Schlumberger-Doll Research
}

$P_{h}$ that is, to date, still widely used, even if there is some disagreement in the literature 10, 11, 12, 13. Within this helical regime, Lubinski and Althouse [5] proposed a loadpitch relationship and Mitchell [14] established that the normal contact force between the helically buckled rod and the cylindrical constraint is quadratically proportional to the applied loads at the end boundaries of the rod. These main results have also been ported to the application of coiled tubing operations, wherein a pipe is injected into a horizontal wellbore, with similar, albeit heterogeneous, behavior observed. For more details on this application, see [15.

The preceding studies were performed under the simplifying assumptions of frictionless rod-constraint interaction and perfect contact along its entire length. The role of frictional drag on the end loads has been considered by Wu and Juvkam-Wold 11, and the effect of end torque has also been investigated [12, 10, 16. Gao and Miska [17, 18. later obtained expressions for the critical sinusoidal and helical loads for the case when lateral friction between the rod and constraint is considered. More recently, Chen et. al [19, 20, 21] have taken into account the contact loss between the rod and the constraint. Beyond perfectly straight constraints, the buckling of a rod inside a cylinder whose long axis has a constant curvature has also been investigated 22, 23. Many studies include experimental confirmation of theoretical results [11, 23, 24, 25, 26. for limited combinations of pipe and constraint diameters. However, to date, there has been no systematic study that 5

Preprint submitted to Extreme Mechanics Letters

January 19, 2015 
behavior depends on geometric and material parameters, as well as test and validate existing theories, in detail.

Here, we perform a joint experimental and numerical investigation of the buckling of a rod inside a cylinder, 60 over a wide range of radial clearances between the rod and the constraint. Our precision experiments are performed on a benchtop-scale apparatus, which allows for the accurate control of the underlying parameters and detailed measurement of the mechanical response. The simulations we use the Kirchhoff rod model to solve for the dynamics of the cylindrically constrained rod. A direct comparison between experiments an simulations, with no fitting parameters, yields good agreement in the force-displacement phenomenology and the frictional drag characteristics, as 70 well as on how the critical loads for sinusoidal and helical buckling depend on radial clearance. We also find that geometric imperfections of the constraint, i.e. deviations from a perfectly straight pipe, have significant effects on the critical loads, compared to theoretical predictions, and 75 that they manifested differently in the cases of sinusoidal and helical buckling.

Our paper is organized as follows. In $\$ 2$, we introduce the experimental apparatus and the corresponding computational framework in $\$ 3$. The combined experimental and 80 numerical results are presented in $\$ 4$, focusing on representative load-displacement curves ( 4.1 , the dependence of the critical loads on the governing parameters (\$4.2) and the effect of imperfection $\$ 4.3$. Finally, in $\$ 5$ we summarize the main results of the paper, present conclusions and ${ }^{115}$ provide a perspective for future work.

\section{Experimental Setup}

In Fig. 1, we present a schematic and photograph of ${ }^{120}$ our apparatus. The experiments consisted of compressing a Nitinol rod inside an acrylic pipe (3.095 m long). The pipe was set horizontal and mounted onto a stiff aluminum frame by five acrylic clamps that were spaced at $75 \mathrm{~cm}$ intervals. Seven different pipes with inner diameter ${ }^{125}$ in the range $D_{i}=3-19 \mathrm{~mm}$ were used. Two Nitinol rods were used with diameter $d=2 r=\{1.14,1.60\} \mathrm{mm}$ and

${ }_{95}$ Young's modulii $E=\{68.67 \pm 0.27,68.05 \pm 0.15\}$ GPa, respectively. For both of these rods, the volumetric density was $\rho=6539 \pm 82 \mathrm{~kg} / \mathrm{m}^{3}$, and the static and dynamic coef- ${ }^{130}$ ficients of friction (between the outer surface of the Nitinol rod and the inner surface of the acrylic constraining pipe) were $\mu_{s}=0.45 \pm 0.07$ and $\mu_{d}=0.30 \pm 0.03$, respectively. Both $\mu_{s}$ and $\mu_{d}$ were measured in the axial direction of the rod/pipe and we assumed that the lateral coefficient ${ }^{135}$ of friction was equal to the axial counterpart. The combination of the various acrylic pipes and rods resulted in 14 experimental radial clearances, $\Delta r$, which refers to the relative confinement of the rod, as shown in Fig. 1 1 a.

The rod was compressed from one of its ends, and along ${ }^{140}$ the axial direction of the constraining pipe, by a worm gear linear actuator controlled by a stepper motor, that pro-

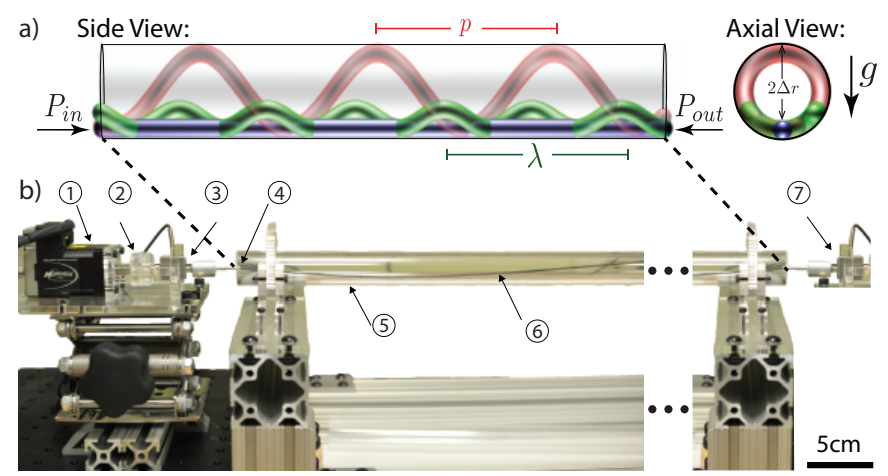

Figure 1: Experimental apparatus. a) Schematic diagram of the configurations of the Nitinol rod inside the horizontal constraining pipe for increasing load. The initially straight rod (blue) first buckles into a sinusoidal configuration (green) with wavelength $\lambda$, and then into a helical configuration (red) with pitch length $p$. The axial view (right) illustrates the radial clearance, $2 \Delta r$, between the rod and the pipe. b) Side view photograph of the experiment: (1) linear actuator, (2) load cells, (3) aluminum pins, (4) acrylic pipe, (5) Nitinol rod, (6) aluminum frame. The Nitinol rod and the acrylic pipe are both $3.095 \mathrm{~m}$ long. For clarity, the photograph has been truncated (marked with ...)

$\mathrm{cm}$. The extremity of the rod near the actuator is referred to as the input end and the other as the output (dead) end. At the input end, a load cell (22.2 N-capacity) was mounted between the linear actuator and an aluminum pin that held the Nitinol rod concentrically within the constraining pipe. The rods used were sufficiently long for the majority of their length to rest along the bottom of the constraint at the beginning of a test. The load cell measured the input load, $P_{i n}$. The aluminum pin was machined to allow the end of the Nitinol rod to rotate with three degrees of freedom while imposing axial position by inserting the rod end into a small (0.3 mm-deep, $1.6 \mathrm{~mm}$ diameter) recess on the pin face. At the output end, the rod was held by an identical aluminum pin which attached to the rigid frame via another load cell, that allowed for measuring the output load, $P_{\text {out }}$.

Each experimental run consisted of first positioning the Nitinol rod inside the horizontal pipe and placing its ends into the two pin recesses. At this stage, we ensured that $P_{\text {in }}$ and $P_{\text {out }}$ were balanced to within $0.4 \mathrm{~N}$ of each other. Loading (compression) was then accomplished by quasistatically increasing the position of the input end in steps of $10 \mu \mathrm{m}$, while recording both $P_{\text {in }}$ and $P_{\text {out }}$. Note that, during a run, $P_{\text {out }}$ was always less than $P_{\text {in }}$ due to frictional losses between the rod and the pipe. Both the stepper motor control and the data acquisition were computer controlled using LabView. A run was stopped when either the maximum travel $(2 \mathrm{~cm})$ of the input end was reached or when the reaction load on either end reached $90 \%$ of the capacity of the load cells. At the end of a run, the system was unloaded by reverse-stepping the linear actuator back to the original position. An experimental test for specific values of the control parameters (e.g. rod or 
pipe diameters) comprised five identical runs to confirm and/or the acrylic pipe were changed for a new geometry, and both the rod and pipe were cleaned with a cotton cloth prior to performing a new test.

\section{Numerical Framework}

Our simulation model uses the shearable and extensible Kirchhoff rod model [27, 28, 29, 30, 31] to describe the elastic rod within the cylindrical constraint. In this framework, the rod is characterized by both its center line position $\boldsymbol{r}(s, t)$, and a material triad $\hat{d}_{i}(s, t)(i=1,2,3)$ at-

155 tached to every cross section that follows the twist along the rod. The arc length of the undeformed rod is $s$ (with $s=0$ at the input end, where the rod is compressed), $t$ is time, $\hat{d}_{1}$ and $\hat{d}_{2}$ lie on the cross section plane whose unit normal is $\hat{d}_{3}$ (for further details on the Kirchhoff rod formulation, see 32]). The four remaining unknowns include the tensile and shear strains, $\gamma(s, t)$ (with $\gamma_{3}=\gamma \cdot \hat{d}_{3}$ representing tensile strain), and the bending and twisting strains, $\boldsymbol{\kappa}(s, t)$ (with $\kappa_{3}=\boldsymbol{\kappa} \cdot \hat{d}_{3}$ denoting the twisting strain). Note that the shear and extensional strains ${ }^{185}$ $\boldsymbol{\gamma}=\partial \boldsymbol{r} / \partial s-\hat{\boldsymbol{d}}_{3}$ can be non-zero since the rod is not assumed to be inextensible nor unshearable. In other words, $\hat{d}_{3}$ is not required to be tangent to the centerline of the rod.

Under these assumptions, the governing equations of the rod are the conservation of linear and angular momentum, respectively,

$$
\begin{array}{r}
\frac{\partial(\rho \boldsymbol{v})}{\partial t}=\frac{\partial(\mathbf{B} \gamma)}{\partial s}+\boldsymbol{F}_{e x t} \\
\frac{\partial(\mathbf{I} \boldsymbol{\omega})}{\partial t}=\frac{\partial(\mathbf{G} \boldsymbol{\kappa})}{\partial s}+\left(\boldsymbol{\gamma}+\hat{d}_{3}\right) \times(\mathbf{B} \gamma)+\boldsymbol{Q}_{e x t}
\end{array}
$$

where $\rho$ is the density, $\boldsymbol{v}=\partial \boldsymbol{r} / \partial t$ is the velocity, $\mathbf{I}$ is the mass moment of inertia tensor, $\boldsymbol{\omega}$ is the angular velocity195 of the material triad, $\mathbf{B}=G A \hat{d}_{1} \hat{d}_{1}+G A \hat{d}_{2} \hat{d}_{2}+E A \hat{d}_{3} \hat{d}_{3}$, so that $\mathbf{B} \gamma$ represents the internal elastic forces; and $\mathbf{G}=$ $E I \hat{d}_{1} \hat{d}_{1}+E I \hat{d}_{2} \hat{d}_{2}+G J \hat{d}_{3} \hat{d}_{3}$, so that $\mathbf{G} \boldsymbol{\kappa}$ represents the internal elastic moments and torques. Here, $E, G, A, I$, and $J$ are the Young's modulus, shear modulus, cross-sectional200 area, second moment of inertia, and polar moment of inertia, respectively. Finally, $\boldsymbol{F}_{\text {ext }}$ and $\boldsymbol{Q}_{\text {ext }}$ are the external applied forces and moment per unit rod reference length.

In order to apply the above Kirchhoff model to our case of a rod compressed inside a cylindrical constraint, we need205 to consider the external forces and moments, namely: i) gravity, ii) normal contact force and iii) friction exerted by the cylindrical wall onto the rod. First, the gravitational force per unit length is

$$
\boldsymbol{F}_{\text {gravity }}=\rho g A \hat{e}_{z},
$$

where $\rho$ is the volumetric density of the rod, $g$ is the acceleration due to gravity and $\hat{e}_{z}$ points vertically downwards.
Second, when rod-channel contact occurs, the normal contact force is in the radial direction of the constraint. It consists of an elastic and an inelastic part $\boldsymbol{F}_{\text {contact }}=$ $-\left(N_{\text {elastic }}+N_{\text {inelastic }}\right) \hat{n}$. The magnitude of the elastic contact force per unit length $N_{\text {elastic }}$ can be determined by the radial penetration, $\delta$, of the rod into the constraint (if penetration occurs):

$$
\delta=B N_{\text {elastic }}\left[\frac{2}{3}+\log \left(\frac{\Delta r}{B N_{\text {elastic }}}\right)\right]+\frac{N_{\text {elastic }}}{k},
$$

where $B$ and $k$ are two constants computed using the geometry of the rod and the constraint, $\Delta r$ is the radial clearance of the rod inside the constraint. The expression for this penetration comes from the Hertzian contact between two cylindrical bodies plus the inherent compliance of the two cross-sections. The magnitude of the inelastic part of the contact force, $N_{\text {inelastic }}$, is determined by the radial velocity of the rod $\boldsymbol{v}_{n}$ at the point of contact:

$$
N_{\text {inelastic }}=C \boldsymbol{v}_{n}
$$

where $C$ is a constant computed using the coefficient of restitution of the contact. Both the elastic and inelastic parts of the contact force act normal to the inner surface of the constraining pipe, if and only if, it is in contact with the pipe wall. More information on the contact model can be found in refs. 33, 34, 35, 36.

Finally, friction is assumed to obey a Coulomb law

$$
\boldsymbol{F}_{\text {friction }} \leq-\mu\left|\boldsymbol{F}_{\text {contact }}\right| \frac{\boldsymbol{v}_{\text {contact }}}{\left|\boldsymbol{v}_{\text {contact }}\right|},
$$

where $\mu$ is the friction coefficient and $\boldsymbol{v}_{\text {contact }}$ is the portion of the velocity vector of the contact point that is tangent to the cylindrical constraint (perpendicular to $\hat{n}$ ). It is important to note that because friction acts on the surface of the rod, instead of at its center, the moment, $\boldsymbol{Q}_{\text {ext }}=$ $r \hat{n} \times \boldsymbol{F}_{\text {friction }}$, emerges and must also be taken into account (no other moments are considered). We also distinguish between static and dynamic friction coefficients, both of which were measured in experiment.

Our simulations solve for the dynamics of the cylindrically constrained Kirchhoff rod model described above, following an approach similar to that previously applied to study the transient dynamics of drillstrings 34, 35, 36. First, the rod is discretized into segments, each of which characterized by its position and orientation. At each computational time step, the extension, bending, twisting, and shear strains are computed for each segment using the current position and orientation of the rod following the Kirchhoff model described above. The stresses along the rod are then computed from these strains, making use of the constitutive law, for which we assume an isotropic linear elastic material. The validity of this assumption is supported by the fact that, given the slenderness of the rod, the material strains remain small throughout the loading process, despite the large displacements and rotations 
rod. From these stresses, we calculate the internal forces acting between neighboring segments that, combined with the external forces (e.g., gravity and the normal/tangential frictional contact forces with constraint), yield the total force acting on each segment. The accelerof the segments can then be determined and, subsequently, the positions updated for the next numerical time step using a Newton-Raphson iteration scheme. The time step $\left(d t=1.6 \times 10^{-6} \mathrm{~s}\right)$ and segment length $(L=2.54 \mathrm{~mm})$ were selected based on a convergence analysis.

\section{Results}

Having introduced our methodology and approach, we proceed with the presentation of our combined experimental and numerical results and a detailed comparison to existing theories. We focus on load-displacement curves 4.1), measure the critical buckling loads $(\$ 4.2)$ and evaluate the importance of geometric imperfections in the buckling process of our system $(\$ 4.3)$.

\subsection{Load-Displacement Curves}

In Fig. 2a we present a representative reaction load versus displacement curve for a Nitinol $\operatorname{rod}(d=1.14 \mathrm{~mm})$ that is compressed axially inside a pipe with $I D=9.4 \mathrm{~mm}$ $(\Delta r=4.1)$ for both experiments (solid symbols) and simulations (hollow symbols). The reaction loads at the $i n$ put end, $P_{\text {in }}$ (circles), and output end, $P_{\text {out }}$ (triangles), of the apparatus are recorded by the load cells on both extremities (see \$2). The two signals are similar up to $\delta \approx 2.5 \mathrm{~mm}$ in the experimental data and $\delta \approx 2 \mathrm{~mm}$ in the simulation, beyond which there is a growing load loss, $\Delta P=P_{\text {in }}-P_{\text {out }}$. This is due the increasing frictional drag between the rod and the surface of the constraining pipe. In the simulations, there is a large peak just prior to sinusoidal buckling, which we do not observe consistently in the experiments. We attribute this difference to the fact that, in the simulations, the rod is perfectly (with its ends laying on the bottom of the constraining pipe), which results in an overshooting of the critical buckling force; a common issue in structural stability algorithms 37.

The region inside the dashed box of Fig. 2 a is a zoom The rod is initially straight and exhibits a reaction load that is both linear and stiff until the first (of two) buckling transitions into the sinusoidal configuration at $\delta_{s}$. After this point, there is a dramatic drop in the slope of the $P-\delta$ curve. lar indicating that there is a nearly full load transfer from the input to the output end. As the rod is compressed further, there is a sudden drop in both $P_{\text {in }}$ and $P_{\text {out }}$ at $\delta_{h}$, when the rod transitions from a sinusoidal into a helical configuration (observed as the rod touches the top of the pipe) and momentarily loses contact with the constraining cylinder during the mode change. Beyond this point, we
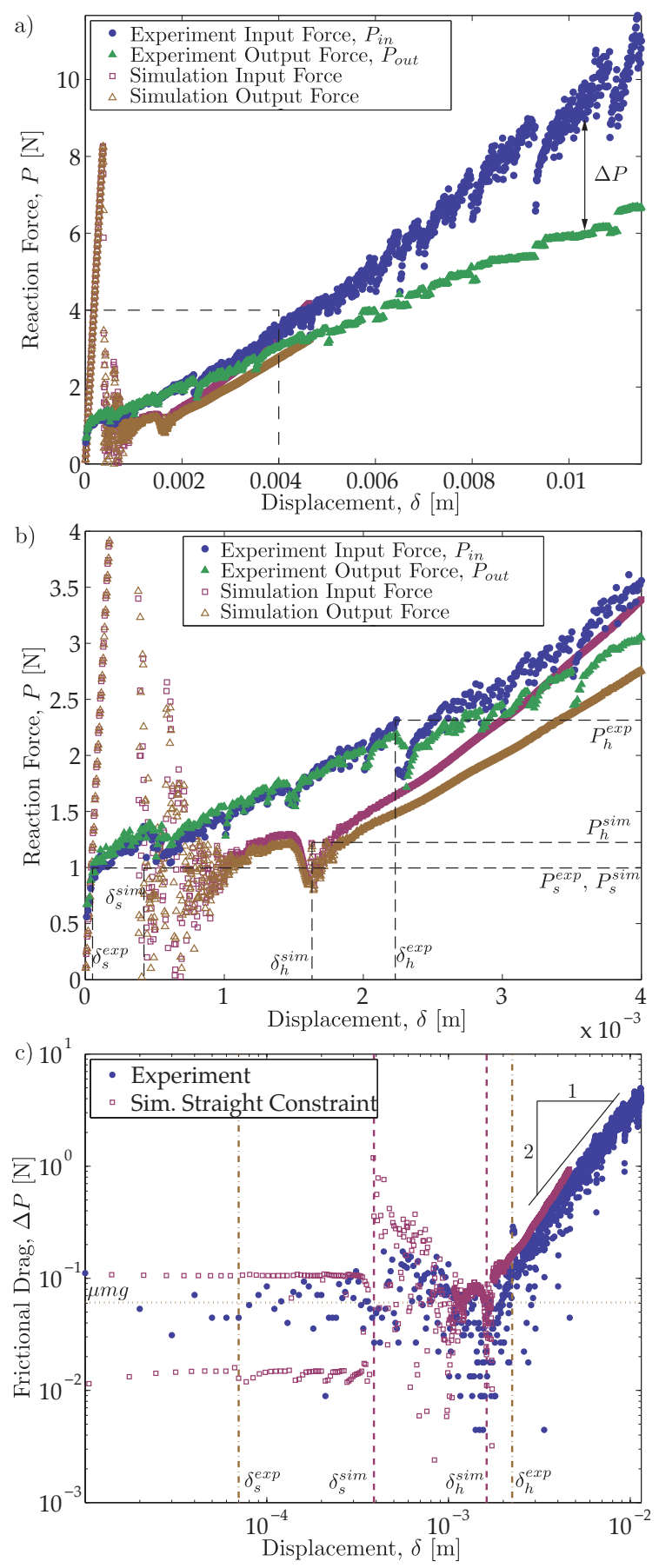

Figure 2: a) Reaction force for experiments (solid symbols) and simulations (hollow symbols) at the input end, $P_{i n}$ (circles), and output end, $P_{\text {out }}$ (triangles), as a function of imposed displacement, $\delta$, for a representative test $(I D=9.4 \mathrm{~mm}, d=1.14 \mathrm{~mm}, \Delta r=4.1 \mathrm{~mm})$. b) Zoom of region inside the dashed box in a), over the range $0<\delta<0.004$. The critical indentations, $\delta_{s}$ and $\delta_{h}$, and critical loads, $P_{s}$ and $P_{h}$, for the transitions between the straight-tosinusoidal and sinusoidal-to-helical configurations, respectively, are marked in the plot for the experimental values. c) Load loss between input and output ends, $\Delta P=P_{\text {in }}-P_{\text {out }}$, as a function of imposed displacement, $\delta$, for experiments (solid symbols) and simulations (hollow symbols). 
find synchronized drops in $P_{\text {in }}$ and $P_{\text {out }}$ that corresponds to the formation of new helical pitches. The helical configtains a number of regions where chirality inverts (known as perversions in the literature of rods [38]), presumably due to the friction-induced frustration of torsional along the length of the pipe. Between $\delta_{s}$ and $\delta_{h}$ in the simula$P_{\text {out }}$, which we attribute to the propagation of elastic axial waves in the simulation, which does not include material damping.

The growing separation between $P_{\text {in }}$ and $P_{\text {out }}$ (Fig. 2 a) normal contact force with the constraint. This causes an increase in frictional drag, which, when integrated over the full length of the rod makes up for $\Delta P$. In Fig. 22, we plot $\Delta P$ versus $\delta$ for experiments (solid circles) and simulations llow circles). We note that this plot is different from previous work [11, 24, 25, which has plotted $\Delta P$ as a function of $P_{i n}$. For imposed displacements below $\delta<\delta_{h}$, the load loss is approximately constant and equal to the sliding friction resistance; $\Delta P \approx \mu_{d} m g$, where $m$ is the we find that $\Delta P \sim \delta^{2}$. This power-law behavior is robust across all experimental tests with other values of $d$ and $\Delta r$. The quadratic dependence between end displacement and normal contact force (which, when combined with friction, theoretical work assumed frictionless interactions between rod and constraint. The $\Delta P \sim W_{n} \sim \delta^{2}$ behavior can therefore be attributed to the nonlinear geometry of the configuration, and the varying axial load in the rod (due to ictional drag) does not affect the functional relationship between displacement and normal contact force.

\subsection{Critical Loads for Sinusoidal and Helical Buckling}

We now turn to studying the loads at which the constrained rod configurations transition from straight to sinusoidal, $P_{s}$, and from sinusoidal to helical, $P_{h}$. In the previous section, we indicated how critical loads were identified by critical points on the $P-\delta$ curves. Motivated by results from the recent literature 6, 9, 17, 18 (discussed in more detail below), loads are normalized by $2 \sqrt{E I w}$ (and denoted by an overbar, e.g., $\bar{P}_{s}=P_{s} /(2 \sqrt{E I / w})$ ), where $E I$ is the bending stiffness of the rod ( $I$ is the second moment of inertia) and $w=\rho g \pi r^{2}$ is its weight per unit length.

In Fig. 3 a,b, we plot the normalized sinusoidal and hefrom experiments (solid symbols) and simulations (hollow symbols), as a function of $\Delta r$, for the two rods with $d=\{1.14,1.6\} \mathrm{mm}$. We also compare the experimental data with two different theoretical predictions from existing literature 6, 9. The simulation results are further subdivided into results with a perfectly axially straight constraining cylinders (hollow circles and squares) and those with geometric imperfections of the constraint that match the experimentally measured values (hollow triangles). The nature of these imperfections will be discussed in detail in $\$ 4.3$.

The first theory (dashed lines in Figs. 3a,b) 6, 9] assumes a frictionless rod-constraint interaction and provides the following predictions for the normalized sinusoidal and helical buckling loads, respectively,

$$
\begin{aligned}
& \bar{P}_{s}(\mu=0)=\frac{P_{s}}{2 \sqrt{E I w}}=\frac{1}{\sqrt{\Delta r}}, \\
& \bar{P}_{h}(\mu=0)=\frac{P_{h}}{2 \sqrt{E I w}}=\sqrt{\frac{2}{\Delta r}} .
\end{aligned}
$$

The second theory (solid line in Figs. 3a,b) does assume lateral friction between the rod and the pipe, and adds an amplification factor $\psi_{c}^{s}$ and $\psi_{c}^{h}$ to Eqs. (7) and (8), respectively, that includes the coefficient of dynamic friction,
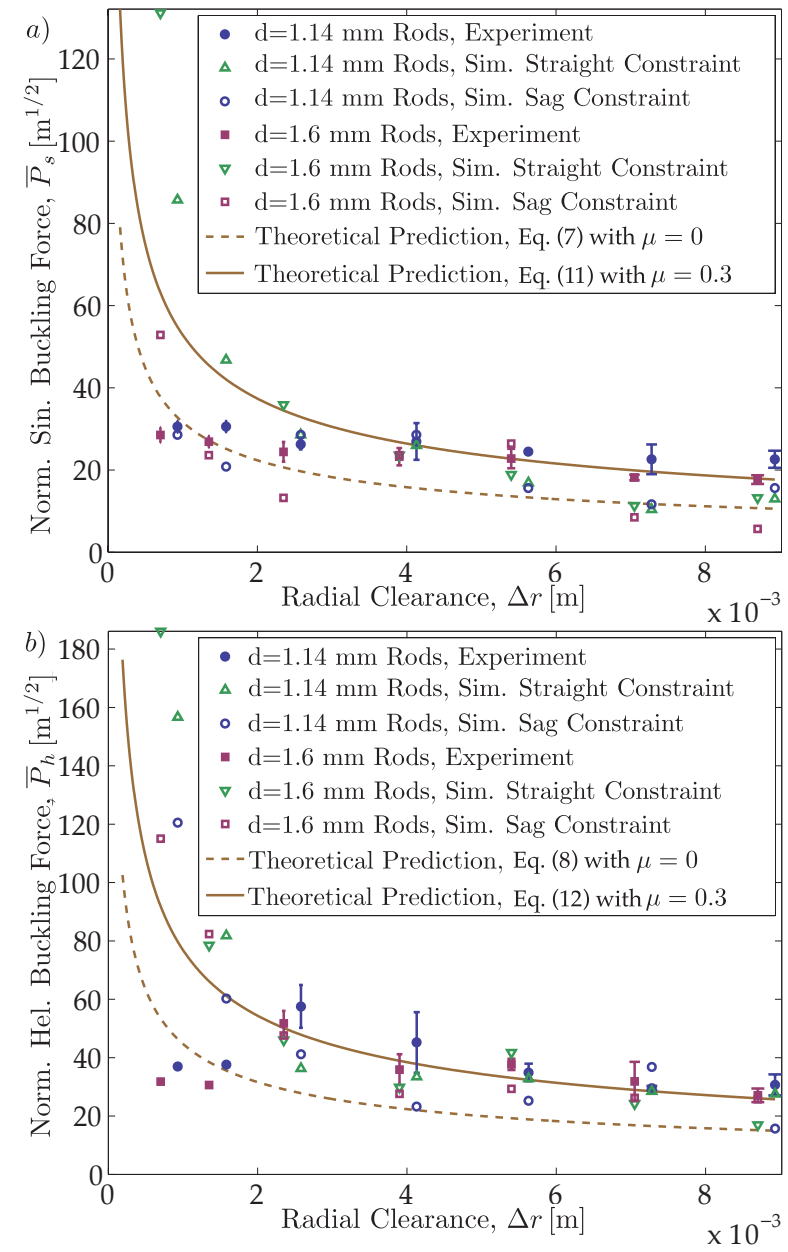

Figure 3: Normalized critical a) sinusoidal and b) helical buckling loads ( $\bar{P}_{s}$ and $\bar{P}_{h}$, respectively) as a function of $\Delta r$. Experimental values are the mean of five runs with error bars representing the standard deviation. These values are compared to theoretical predictions from the literature for the case of frictionless (dashed line) and frictional (solid line) interactions between the rod and constraint 6. 9 . 
$\mu_{d}[17,18$ :

$$
\begin{gathered}
\psi_{c}^{s}=\frac{q^{2}}{2}\left(1-1.5 a^{2}\right)+\frac{1}{2 q^{2}}\left(1+\frac{a^{2}}{8}+\frac{8 \mu_{d}}{\pi a}\right), \\
\psi_{c}^{h}=\frac{6}{3-\pi \mu_{d}}\left(\frac{\left(\pi+2 \mu_{d}\right)\left(5-\pi \mu_{d}\right)}{10 \pi}\right)^{1 / 4},
\end{gathered}
$$

where $q=1+0.193 \mu_{d}^{2 / 3}$ and $a=0.774 \mu_{d}^{1 / 3}-0.371 \mu_{d}$. For our measured value of $\mu_{d}=0.30 \pm 0.03$ and pinned-pinned boundary conditions, $\psi_{c}^{s}=1.67$ and $\psi_{c}^{h}=2.44$. In Fig. 3a,b we plot (solid lines):

$$
\begin{aligned}
& \bar{P}_{s}\left(\mu=\mu_{d}\right)=\frac{P_{s}}{2 \sqrt{E I w}}=\frac{\psi_{c}^{s}}{\sqrt{\Delta r}}, \\
& \bar{P}_{h}\left(\mu=\mu_{d}\right)=\frac{P_{h}}{2 \sqrt{E I w}}=\frac{\psi_{c}^{h}}{\sqrt{\Delta r}} .
\end{aligned}
$$

340 The two experimental datasets $(d=\{1.14,1.6\} \mathrm{mm})$ collapse onto a single curve, for both $\bar{P}_{s}$ and $\bar{P}_{h}$, which supports the chosen normalization. For large values of the radial clearances, these experimental results for $\bar{P}_{s}$ and $\bar{P}_{h}$ agree particularly well with Eqs. (11) and (12), which both370 however, there is a pronounced disagreement and the frictional theory significantly overpredicts the experimental data $\left(\sim 50 \%\right.$ for $\bar{P}_{s}$ and $\sim 100 \%$ for $\left.\bar{P}_{h}\right)$. Simulation data for perfectly straight clearances show a similar collapse,375 and agree well with Eqs. (11) and (12) over all clearances, with critical forces slightly higher than those predicted. On the other hand, when geometric imperfections of the constraint are included in the simulations (to match the experiments), significant discrepancies with the predictions380 are found, similarly to the experimental results. The role of the geometrical imperfections of the setup in affecting the mechanical response of our system will be discussed in more detail, below, in $\$ 4.3$

\subsection{Effect of Imperfections}

As described in $\$ 2$, the acrylic pipe used in our experimental apparatus was mounted horizontally to a rigid aluminum frame at discrete points set $75 \mathrm{~cm}$ apart (see Fig. 11. These unsupported segments can sag under their own weight. Assuming clamped-clamped boundary conditions, the maximum deflection due to self-weight at the mid-span is 39

$$
\varsigma_{\max }=\frac{w L^{2}}{384 E I}
$$

where $L$ is the unsupported length of the pipe between clamps, $w=1195 \mathrm{~kg} / \mathrm{m}^{3}$ is weight per unit length of the glass pipe, $E=2.2 \mathrm{GPa}$ is its Young's modulus and $I=$ $\pi / 64\left(D_{o}^{4}-D_{i}^{4}\right)$ is the area moment of inertia of the pipe (which we vary for pipes with different outer and inner di-395 ameters, $D_{o}$ and $D_{i}$, respectively). For convenience, we define the dimensionless maximum deflection, $\eta=\varsigma_{\max } / \Delta r$ such that $\eta=0$ corresponds to the pipe being perfectly straight and $\eta>1$ to a deflection that is larger than the radial clearance. In Fig 4 , we plot $\eta$ versus $\Delta r$ for all 400

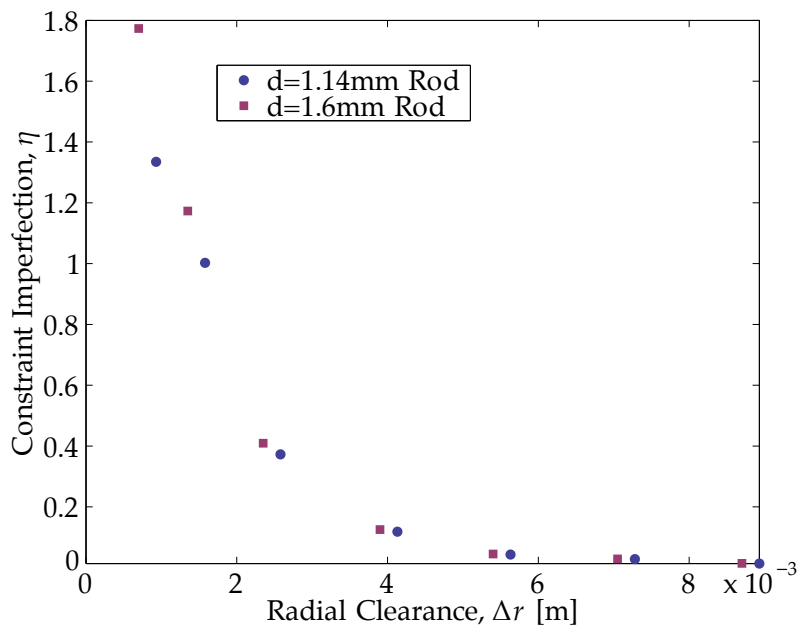

Figure 4: Normalized maximum sag in the constraint, $\eta$, as a function of the radial clearance, $\Delta r$, for all the experimental runs.

the acrylic pipes used in our experiments, and for the two cases of $d=\{1.15,1.6\} \mathrm{mm}$. Note that $\eta \gtrsim 1$ for the two tightest clearances, the third tightest clearance has $\eta \approx 0.4$ and $\eta \leq 0.05$ for $\Delta r \gtrsim 4 \mathrm{~mm}$.

We revisit the critical buckling loads plotted in Fig. $3 \mathrm{a}, \mathrm{b}$ that showed disagreement between experimental results and frictional theory at lower clearances, which we now understand as having larger values of $\eta$. As noted, across the whole range of $\Delta r$, simulations for a straight constraint follow Eq. 12 , whereas the simulations with a geometrically imperfect pipe follow the experiments more closely.

We further evaluate the deviations of the experimental and simulated (including sag) data from Eq. (12) by quantifying the following normalized differences between the theoretical prediction with friction from Eq. (11) and Eq. $12,,\left[P_{s}\right]_{t h}$ and $\left[P_{h}\right]_{t h}$, and the observed experimental and simulated results, $\left[P_{s}\right]_{o b s}$ and $\left[P_{h}\right]_{o b s}$, for the critical buckling loads for the onset of the sinusoidal and buckling configuration, respectively,

$$
\begin{aligned}
\chi^{s} & \equiv \frac{\left[P_{s}\right]_{t h}-\left[P_{s}\right]_{o b s}}{\left[P_{s}\right]_{o b s}}, \\
\chi^{s} & \equiv \frac{\left[P_{h}\right]_{t h}-\left[P_{h}\right]_{o b s}}{\left[P_{h}\right]_{o b s}} .
\end{aligned}
$$

These can be regarded as knockdown factors, as often referred to in buckling analysis, with $\chi=0$ indicating perfect agreement between experiments and theory.

In Fig. $5 \mathrm{a}$ and $\mathrm{b}$, we present $\chi^{s}$ and $\chi^{h}$ versus $\eta$, for the two rods with $d=\{1.14,1.6\} \mathrm{mm}$. The knockdown factor is approximately linear with imperfection size. Negative values of $\chi^{s, h}$ correspond to observed measurements greater than theoretical predictions. It is interesting to note that for the case of $\chi^{h}$, there is a threshold of $\eta \sim 0.4$, below which the system is insensitive to imperfections. This indicates that there is a range, $0<\eta \lesssim 0.4$, over which the system is tolerant to the size of the imperfec- 

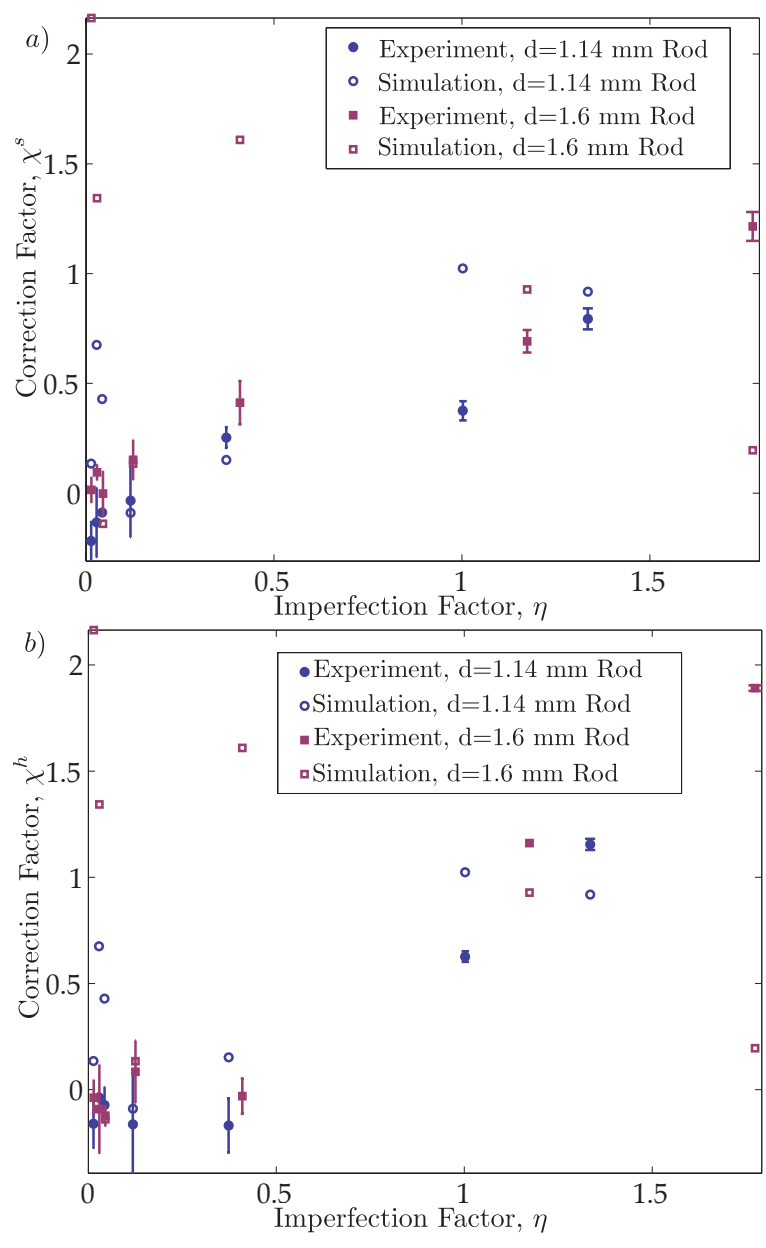

Figure 5: Experimental deviation from theoretical prediction for crit- 450 ical a) sinusoidal and b) helical buckling load ( $\chi^{s}$ and $\chi^{h}$, respectively) as a function of constraint imperfection size, $\eta$. We note an approximately linear relationship between $\chi^{s}$ and $\eta$ in a), but an apparent critical value of $\eta\left(0.4<\eta_{c}<1\right)$ in b) below which $\chi^{h}$ remains relatively unaffected.

tions on the constraining geometry, where the idealized frictional theory [17] mentioned above is applicable. Beyond this range, however, reductions in the critical condi- ${ }^{460}$ tions for the onset of helical buckling can be significant, by as much as $300 \%$ (see Fig. 5 ).

\section{Conclusions}

We have studied the problem of buckling of a cylindrically constrained rod under compression, through a com- ${ }_{470}$ bination of precision model experiments and simulations, finding good agreement between the two, with no fitting physical parameters. These results were also compared to existing theoretical predictions that describe the data well ${ }_{475}$ for the larger radial clearances, but significant discrepancies were found for tighter clearances. Exploring these differences led to the recognition of the nontrivial role of geometric imperfections of the horizontal constraint, no- ${ }_{480}$ tably the sag that was present in the unsupported spans of the smaller-diameter pipes. We found that the knockdown factor that reconciles our results with existing theory increased with the size of the imperfection (compared to the radial clearance). For the onset of the helical configuration, however, there was a critical threshold below which the system is insensitive to imperfections. This finding should be particularly relevant for coiled-tubing operations in horizontal wellbores with an intrinsic tortuosity, where there is a timely need to extend reach [40. To the best of our knowledge, the importance of this knockdown (and the existence of a threshold) has not previously been reported in the literature for the context of coiled-tubing imperfection-dependence for different wavelengths of imperfection size, as well as imperfections not aligned with the direction of gravity. and are grateful for financial support from both Schlumberger and the National Science Foundation (CMMI-1129894).

\section{References}

[1] W. Klug, M. Feldmann, M. Ortiz, Comp. Mech. 35 (2005) 146152.

[2] F. Xu, W. Lu, Y. Zhu, ACS Nano 5 (2010) 672-678.

[3] D. Svenšek, R. Podgornik, Phys. Rev. E 77 (2008) 031808.

[4] J. L. Silverberg, R. D. Noar, M. S. Packer, M. J. Harrison, C. L. Henley, I. Cohen, S. J. Gerbode, P. Natl. A. Sci. 109 (2012) 16794-16799.

[5] A. Lubinski, W. Althouse, J. Petrol. Technol. 14 (1962) 655670.

[6] P. Paslay, D. Bogy, J. Appl. Mech. 31 (1964) 605-610.

[7] A. Lubinski, H. Woods, Drilling and production practice (1953) $222-250$

[8] R. Dawson, P. Paslay, J. Petrol. Technol. 36 (1984) 1734-1738.

[9] Y. Chen, Y. Lin, J. Cheatham, J. Petrol. Technol. 42 (1990) 140-141.

[10] G. Deli, F. Liu, B. Xu, in: SPE International Oil and Gas Conference and Exhibition in China, pp. 517-523.

[11] J. Wu, H. Juvkam-Wold, in: SPE Production Operations Symposium, pp. 867-876.

[12] S. Miska, J. Cunha, in: SPE Production Operations Symposium, pp. 173-180.

[13] S. Miska, W. Qiu, L. Volk, J. Cunha, in: International Conference on Horizontal Well Technology, pp. 207-214.

[14] R. Mitchell, SPE Drilling Engineering 1 (1986) 457-465.

[15] J. T. Miller, Mechanical behavior of elastic rods under constraint, Ph.D. thesis, Massachusetts Institute of Technology, 2014.

[16] W. Qiu, in: SPE Eastern Regional Meeting, pp. 329-342.

[17] G. Gao, S. Miska, SPE J. 14 (2009) 782-796.

[18] G. Gao, S. Miska, SPE J. 15 (2010) 1104-1118.

[19] J.-S. Chen, H.-C. Li, J. Appl. Mech. 78 (2011).

[20] J. Fang, S.-Y. Li, J.-S. Chen, Acta Mech. 224 (2013) 2635-2647.

[21] S.-Y. Li, J.-S. Chen, Eur. J. Mech. A - Solid. 44 (2014) 61-74.

[22] X. He, A. Kyllingstad, SPE Drill. Completion 10 (1995) 10-15.

[23] E. Kuru, A. Martinez, S. Miska, W. Qiu, in: SPE/IADC Drilling Conference, pp. 1-9.

[24] R. McCann, P. Suryanarayana, in: Offshore Technology Conference, pp. 511-521.

25] P. Suryanarayana, R. McCann, in: Presentation given at the ASME Energy-Source Technology Conference and Exhibition, New Orleans, pp. 23-27.

[26] A. Martinez, S. Miska, E. Kuru, J. Sorem, J. Energ. Resour. ASME 122 (2000) 123-128. 
[27] G. Kirchhoff, Journal für die Reine und Angewandte Mathematik 56 (1859) 285-313.

28] A. Love, A treatise on the mathematical theory of elasticity, first ed., Cambridge University Press, 1892.

[29] A. Clebsch, Theorie der elasticitat fester korper, BG Teubner, 1862.

[30] J. H. Maddocks, Arch. Ration. Mech. An. 85 (1984) 311-354.

[31] S. S. Antman, Nonlinear problems of elasticity, volume 107, Springer, 2005.

[32] B. Audoly, Y. Pomeau, Elasticity and geometry: from hair curls to the non-linear response of shells, Oxford University Press Oxford and New York, 2010.

[33] R. J. Roark, W. C. Young, Formulas for stress and strain, McGraw-Hill, 1975.

[34] J. Pabon, N. Wicks, Y. Chang, R. Harmer, Non-linear Dynamics of Deep Drilling Systems (2009) 83.

[35] J. A. Pabon, N. Wicks, Y. Chang, B. Dow, R. J. Harmer, et al., in: SPE Deepwater Drilling and Completions Conference, Society of Petroleum Engineers, pp. 293-307.

[36] J. Pabon, N. Wicks, Y. Chang, C. Chapman, V. Singh, Modeling the transient behavior of bha/drill string while drilling, Patent, 2011. URL: http://www.freepatentsonline. com/8014987.html

[37] H. M. Hilber, T. J. Hughes, Earthq. Eng. Struct. D. 6 (1978) 99-117.

[38] A. Goriely, M. Tabor, Phys. Rev. Lett. 80 (1998) 1564-1567.

[39] S. Timoshenko, J. Gere, Theory of Elasticty Stability, second ed., Dover, 1961.

510 [40] J. Miller, C. G. Mulcahy, J. Pabon, N. Wicks, P. M. Reis, J. Appl. Mech. 82 (2015) 021003. 